\title{
Genetic Diversity and Population Structure of Rubus Accessions Using Simple Sequence Repeat Markers
}

\author{
Kyung Jun Lee ${ }^{1}$, Gi-An Lee ${ }^{1}$, Hee-Kyoung Kang ${ }^{2}$, Jung-Ro Lee ${ }^{1}$, Sebastin Raveendar, Myoung-Jae Shin ${ }^{1}$, \\ Yang-Hee Cho', Kyung-Ho $\mathrm{Ma}^{1}$ * \\ ${ }^{1}$ National Agrobiodiversity Center, NAS, RDA, Jeonju 54874, Korea \\ ${ }^{2}$ Department of Horticulture, College of Industrial Science, Kongju National University, Yesan 32439, Korea
}

\begin{abstract}
Sixty-nine Rubus accessions were analyzed to determine the genetic relationships using simple sequence repeat (SSR) markers. Twenty-three SSR markers generated a total of 351 alleles from all accessions, with an average of 15.3 alleles per locus. The average value of polymorphism information content was 0.76 , ranging from 0.52 to 0.91 . As a result of population structure analysis, 69 Rubus accessions of six Rubus species were subdivided into six subpopulations. Four subpopulations included distinct Rubus species accessions; pop2 (Rubus crataegifolius var. subcuneatus, 2 accessions), pop3 (R. crataegifolius Bunge., 18 accessions), pop4 (R. fruticosus L., 3 accessions) and pop6 (R. coreanus Miq., 36 accessions) while The pop1 and pop5 mainly included $R$. idaeus L. and $R$. parvifolius L., respectively. In cluster analysis, 69 Rubus accessions were divided into three groups. Group I contained 35 Rubus accessions, which consisted of six Rubus species. Groups II and III had 30 and 4 Rubus accessions, respectively. They consisted of only $R$. coreanus. The uncertain diversity of species and artificial groups of the Rubus genus has created confusion with respect to the correct classification of the species at both commercial and scientific levels. The results of the present study will provide basic information for phylogeny, taxonomy and breeding programs of Rubus species.
\end{abstract}

Keywords Genetic diversity, Rubus species, Simple sequence repeat, STRUCTURE

\section{INTRODUCTION}

Rubus (Rosaceae) is a large genus including diverse species and is grown extensively worldwide at altitudes ranging from 0 to 4,500 $\mathrm{m}$ above sea level (Marulanda et al. 2012a). Among the flowering plant species, Rubus genus is reported to contain approximately 800 species due to biological processes such as hybridization, apomixis, and polyploidy which weaken species boundaries (Thompson 1995; Alice and Campbell 1999). Rubus has been divided into 12 subgenera; subgenera Idaeobatus, Dalibarda, and Anoplobatus are predominantly diploid, whereas Dalibardastrum, Malachobatus, and Orobatus are exclusively polyploid (Thompson 1995; Thompson 1997). Among the Rubus genus, only a few species such as raspberries, blackberries, dewberries, arctic fruits and flowering raspberries have been domesticated and utilized in breeding programs (Jennings 1988).

Since the distinction and characterization of Rubus genetic resources using morphological markers is difficult because of complexity of the Rubus genus, molecular markers might be reliable means for cultivar identification and assessment of genetic relatedness and diversity in these collections (Castillo et al. 2010). Several studies of the Rubus species have used molecular markers such as amplified fragment length polymorphism (AFLP) (Graham et al. 2004), internal transcribed spacer (ITS) (Alice and Campbell 1999), Random amplification of polymorphic DNA (RAPD) (Graham et al. 1997), and simple sequence repeat (SSR) (Graham et al. 2004; Dossett et al. 2012; Marulanda et al. 2012b) to assess the genetic diversity (GD) and population structures.

Microsatellite loci present a relatively high frequency in the eukaryotic genome and show high reproducibility and

Received July 27, 2016; Revised August 18, 2016; Accepted August 18, 2016; Published August 31, 2016

*Corresponding author Kyung-Ho Ma, khma@korea.kr, Tel: +82-63-238-4870, Fax: +82-63-238-4859 
polymorphism as a codominant molecular marker. Due to these properties, microsatellite markers have been widely used for GD and population analysis in diverse crops (Feingold et al. 2005; Laurent et al. 2007; Lee et al. 2015). Especially for the Rubus species, Lewers et al. (2008) developed microsatellite markers from an expressed sequence tag library of blackberry (Rubus L.) cultivars, and Castillo et al. (2010) isolated twelve microsatellite markers from enriched genomic libraries of red raspberry (Rubus idaeus) and blackberry ( $R$. hybrid). The use of these markers made it possible to analyze the genetic constitution of the hybrids and to perform further phylogenetic and evolutionary studies in Rubus (Alice 2002).

Recently, our lab reported 97 novel microsatellite markers acquired from $R$. coreanus, and tested cross-amplification in other Rubus species (Lee et al. 2015). The aim of the current study is to survey the polymorphism of the previously reported microsatellite markers in diverse Rubus accessions, and to evaluate the distribution of genetic variability and population structure of conserved Rubus diversity.

\section{MATERIALS AND METHODS}

\section{Plant materials}

Sixty-nine Rubus accessions obtained from Gochang
Blackraspberry Research Institute (GBRI), Republic of Korea were used for DNA extraction and further analysis. Plants of the following species were included: $R$. coreanus (36 accessions), $R$. crataegifolius (18 accessions), $R$. idaeus (5 accessions), $R$. fruticosus (4 accessions), $R$. crataegifolius var. subcuneatus (3 accessions), and $R$. parvifolius (3 accessions) (Table 1).

\section{DNA extraction and assessment using microsatellite markers}

Genomic DNA was extracted from bulked samples using Plant DNAzol reagent (Invitrogen, Carlsbad, CA, USA) following the instructions provided by the manufacturer. The DNA concentration was determined using a NanoDrop (ND-1000; NanoDrop, Wilmington, DE, USA). The final concentration of each DNA sample was adjusted to $20 \mathrm{ng} / \mu \mathrm{l}$. The M13-tail at the 5'-end region with the polymerase chain reaction (PCR) method was used to measure the size of the amplified products (Schuelke 2000). Genotyping was carried out using 23 SSR markers listed in Table 2. A set of the polymorphic SSR markers was obtained from the data previously reported by Lee et al. (2015). Amplified fluorescent-labeled PCR products were analyzed on an ABI-Prism 3130x1 Genetic Analyzer (Applied Biosystems, Foster City, CA, USA). Fragments were sized and scored into alleles using GeneMapper v4.0 (Applied Biosystems).

Table 1. List of 69 accessions belonging to the genus Rubus in this study.

\begin{tabular}{|c|c|c|c|c|c|c|}
\hline \multirow{2}{*}{$\begin{array}{r}\text { Rubus species } \\
\text { Rubus coreanus Miq. }\end{array}$} & \multicolumn{6}{|c|}{ Accession number } \\
\hline & GCB0021 & GCB0030 & GCB0038 & GCB0045 & GCB0052 & GCB0061 \\
\hline & GCB0022 & GCB0032 & GCB0040 & GCB0046 & GCB0054 & GCB0062 \\
\hline & GCB0023 & GCB0033 & GCB0041 & GCB0047 & GCB0055 & GCB0063 \\
\hline & GCB0024 & GCB0034 & GCB0042 & GCB0049 & GCB0057 & GCB0064 \\
\hline & GCB0027 & GCB0035 & GCB0043 & GCB0050 & GCB0059 & GCB0118 \\
\hline & GCB0029 & GCB0036 & GCB0044 & GCB0051 & GCB0060 & GCB0119 \\
\hline \multirow[t]{3}{*}{ R. crataegifolius Bunge. } & GCB0006 & GCB0009 & GCB0012 & GCB0015 & GCB0018 & GCB0129 \\
\hline & GCB0007 & GCB0010 & GCB0013 & GCB0016 & GCB0019 & GCB0130 \\
\hline & GCB0008 & GCB0011 & GCB0014 & GCB0017 & GCB0127 & GCB0131 \\
\hline R. crataegifolius var. subcuneatus & GCB0001 & GCB0002 & GCB0120 & & & \\
\hline R. fruticosus L. & GCB0065 & GCB0067 & GCB0068 & GCB0069 & & \\
\hline R. idaeus L. & GCB0071 & GCB0072 & GCB0073 & GCB0074 & GCB0075 & \\
\hline R. parvifolius L. & GCB0003 & GCB0004 & GCB0005 & & & \\
\hline
\end{tabular}


Table 2. List of simple sequence repeat primers used for genotyping of 69 Rubus materials.

\begin{tabular}{|c|c|c|c|c|c|c|c|c|}
\hline Primer & Accession No. & R-motif & Size (bp) & $\mathrm{NA}^{\mathrm{z})}$ & GD & Ho & PIC & MAF \\
\hline GB-RC-074 & JX976555 & $(\mathrm{AG}) 15$ & $138-236$ & 14 & 0.75 & 0.50 & 0.72 & 0.40 \\
\hline GB-RC-077 & JX976557 & $(\mathrm{AAC}) 9$ & $190-212$ & 10 & 0.55 & 0.48 & 0.53 & 0.66 \\
\hline GB-RC-078 & JX976558 & (GCA)6 & $156-252$ & 17 & 0.87 & 0.81 & 0.86 & 0.22 \\
\hline GB-RC-091 & JX976559 & $(\mathrm{AG}) 15$ & $235-271$ & 17 & 0.87 & 0.46 & 0.85 & 0.25 \\
\hline GB-RC-098 & JX976560 & $(\mathrm{GCA}) 4$ & $201-222$ & 6 & 0.57 & 0.29 & 0.52 & 0.61 \\
\hline GB-RC-100 & JX976561 & $(\mathrm{AG}) 6$ & $210-322$ & 24 & 0.91 & 0.40 & 0.90 & 0.19 \\
\hline GB-RC-105 & JX976562 & $(\mathrm{GT}) 8$ & $141-183$ & 13 & 0.77 & 0.78 & 0.75 & 0.42 \\
\hline GB-RC-109 & JX976563 & (TC)12 & $165-205$ & 20 & 0.86 & 0.47 & 0.85 & 0.25 \\
\hline GB-RC-111 & JX976564 & $(\mathrm{CA}) 11$ & $166-248$ & 11 & 0.65 & 0.67 & 0.62 & 0.56 \\
\hline GB-RC-138 & JX976565 & (ACACTC) 4 & $199-227$ & 15 & 0.79 & 0.47 & 0.76 & 0.34 \\
\hline GB-RC-141 & JX976566 & (GA)6 & $163-225$ & 18 & 0.65 & 0.42 & 0.64 & 0.58 \\
\hline GB-RC-145 & JX976568 & (AC)9,(CAA)5 & $256-294$ & 8 & 0.61 & 0.19 & 0.55 & 0.55 \\
\hline GB-RC-146 & JX976569 & $(\mathrm{CTT}) 5$ & $158-194$ & 9 & 0.63 & 0.25 & 0.58 & 0.54 \\
\hline GB-RC-166 & JX976570 & (TTGAAG)6 & $167-213$ & 18 & 0.87 & 0.50 & 0.86 & 0.19 \\
\hline GB-RC-167 & JX976571 & (TC)17 & $187-267$ & 26 & 0.91 & 0.82 & 0.91 & 0.17 \\
\hline GB-RC-178 & JX976572 & $(\mathrm{CA}) 15$ & $151-211$ & 19 & 0.88 & 0.74 & 0.87 & 0.24 \\
\hline GB-RC-186 & JX976573 & (TTG)5 & $267-305$ & 17 & 0.79 & 0.67 & 0.77 & 0.36 \\
\hline GB-RC-191 & JX976574 & (GA)6 & 271-309 & 14 & 0.88 & 0.77 & 0.87 & 0.17 \\
\hline GB-RC-193 & JX976575 & (GA)18 & $273-295$ & 12 & 0.86 & 0.74 & 0.85 & 0.21 \\
\hline GB-RC-194 & JX976576 & $(\mathrm{ACA}) 8$ & $237-261$ & 9 & 0.70 & 0.54 & 0.66 & 0.49 \\
\hline GB-RC-211 & JX976578 & (GGA)7 & $188-210$ & 12 & 0.84 & 0.45 & 0.82 & 0.33 \\
\hline GB-RC-243 & JX976581 & $(\mathrm{TGT}) 9$ & $156-248$ & 21 & 0.81 & 0.49 & 0.79 & 0.32 \\
\hline GB-RC-245 & JX976582 & $(\mathrm{AG}) 14$ & $250-300$ & 21 & 0.92 & 0.70 & 0.91 & 0.13 \\
\hline \multirow{2}{*}{\multicolumn{4}{|c|}{$\begin{array}{r}\text { Total } \\
\text { Mean }\end{array}$}} & 351 & - & - & - & - \\
\hline & & & & 15.3 & 0.78 & 0.55 & 0.76 & 0.35 \\
\hline
\end{tabular}

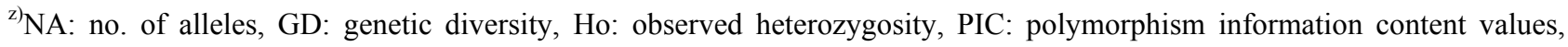
MAF: major allele frequency.

\section{Data analyses of GD}

The total number of alleles, allele frequency, gene diversity and polymorphism information content (PIC) per individual SSR locus were calculated with the PowerMarker version 3.25 (Liu and Muse 2005). The unweighted pair group method with an arithmetic mean (UPGMA) dendrogram was constructed to visualize the phylogenetic distribution of accessions using the software MEGA version 5.03 (Tamura et al. 2007). Principal co-ordinate analysis (PCoA) in GenAlEx 6.5 was used to further examine the genetic relationships among the detected populations on the basis of the SSR data (Peakall and Smouse 2012). STRUCTURE software (V2.3.4) was used to detect the possible subpopulations ( $\mathrm{K}=1$ to $\mathrm{K}=10$ ) with a model allowing for admixture and correlated allele frequencies using a burn-in period of 50,000 and MCMC repeats of 50,000 followed by three iterations (Pritchard et al. 2000). The optimal number of populations corresponds to the highest peak in the $\Delta \mathrm{K}$ graph and the accessions with membership probabilities $\geq 70 \%$ were assigned to each subpopulation (Evanno et al. 2005).

\section{RESULTS}

The 23 SSR markers produced 351 alleles and the number of detected alleles over all loci across genotypes ranged from 6 (GB-RC-098) to 24 (GR-RC-167), with an average of 15.3 alleles per locus (Table 2). With respect to the indices for comparing genetic variability, we calculated PIC and GD values for the used SSR primers; PIC value 
ranged from 0.52 (GB-RC-098) to 0.91 (GB-RC-167 and GB-RC-245), with an average value of 0.76 and GD value ranged from 0.55 (GB-RC-077) to 0.92 (GB-RC-245), with an average value of 0.78 .

The distribution of genetic variation among and within the six populations of accessions was estimated by analysis of molecular variance (AMOVA), which revealed that $26 \%$ of total variation was among populations, and $56 \%$ of variation was within individuals (Table 3). PCoA with SSR markers showed that large diversity existed in Rubus species. The first three axes explained $31.7 \%$ of cumulative variation. The exhibited Rubus genotypes were grouped into four broad groups across the two axes (Fig. 1). The genotypes belonging to the species $R$. coreanus and $R$. crataegifolius were separate from the other species.

To study the population structure, a model-based STRUCTURE was used to determine the genetic relationship among individual Rubus accessions (Fig. 2A). This model assumes that the number of populations was $\mathrm{k}$ and the loci were independent and in Hardy-Weinberg equilibrium. $\mathrm{K}=1$ to 10 populations were tested, $\mathrm{Ln}(\mathrm{PD})$ kept on increasing with increasing population number but there was no clear population structure, therefore $\mathrm{Ln}(\mathrm{PD})$ derived $\Delta \mathrm{k}$ was plotted against the $\mathrm{K}$ to determine the number of populations. At $\mathrm{K}=6$, maximum $\Delta \mathrm{k}$ was found and this was considered as the number of populations for 69 Rubus accessions. Pop-1 included six accessions, pop-2 included two accessions, pop-3 included 18 accessions,

Table 3. Summary analysis of molecular variance for simple sequence repeat markers.

\begin{tabular}{lrrrrr}
\hline \multicolumn{1}{c}{ Source } & \multicolumn{1}{c}{ SS } & \multicolumn{1}{c}{ MS } & Est. Var. & $\%$ \\
\hline Among species & 5 & 287.203 & 57.441 & 2.688 & 26 \\
Among accessions & 63 & 592.906 & 9.411 & 1.847 & 18 \\
Within accessions & 69 & 394.500 & 5.717 & 5.717 & 56 \\
Total & 137 & 1274.609 & & 10.253 & 100 \\
\hline
\end{tabular}

${ }^{\mathrm{z})} \mathrm{df}$ : degree of freedom, SS: sum of square, MS: mean square, Est. Var.: estimate variance.

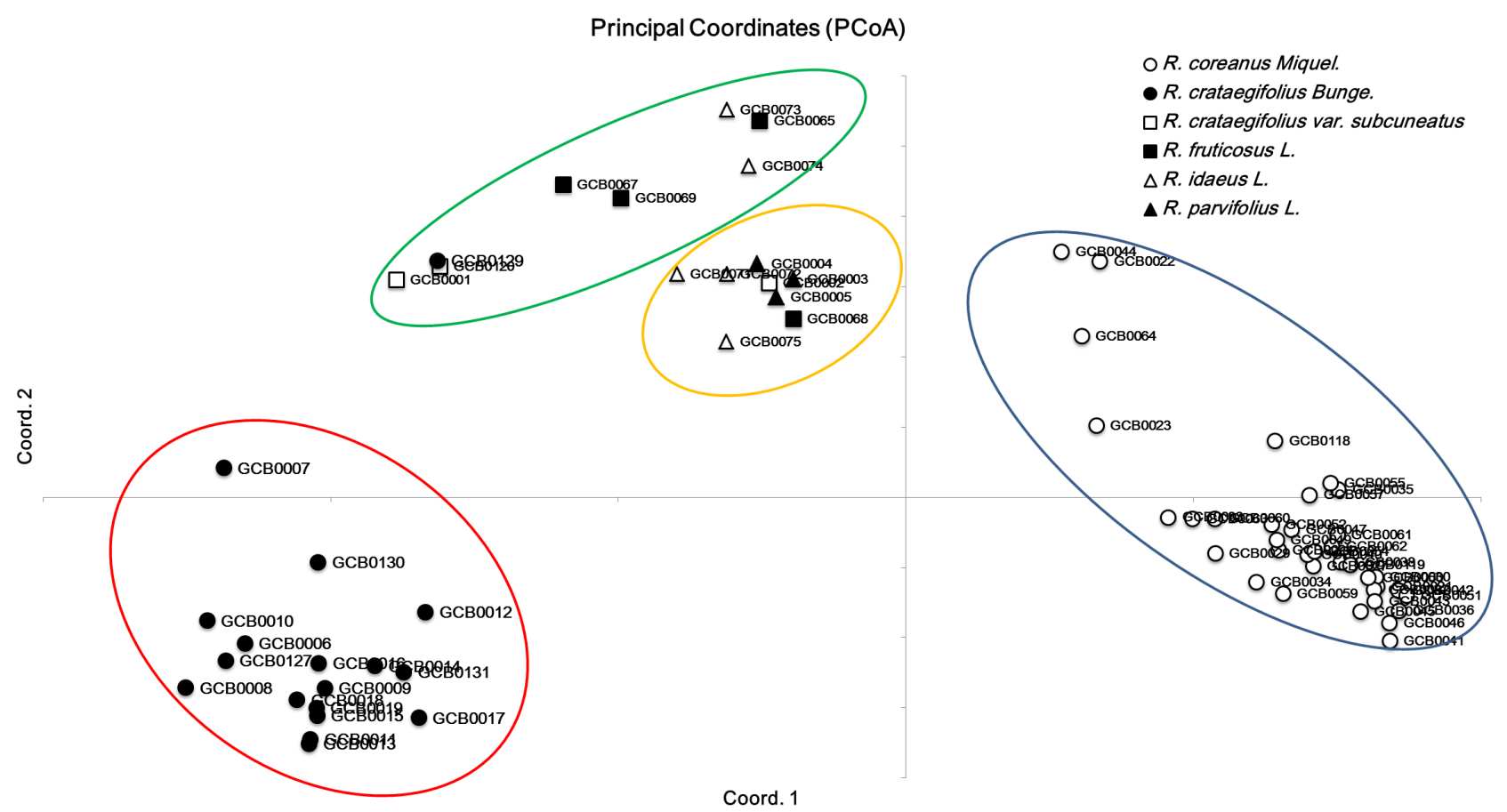

Fig. 1. Principal coordinates analysis among Rubus genotypes based on genetic distance. 
(A)
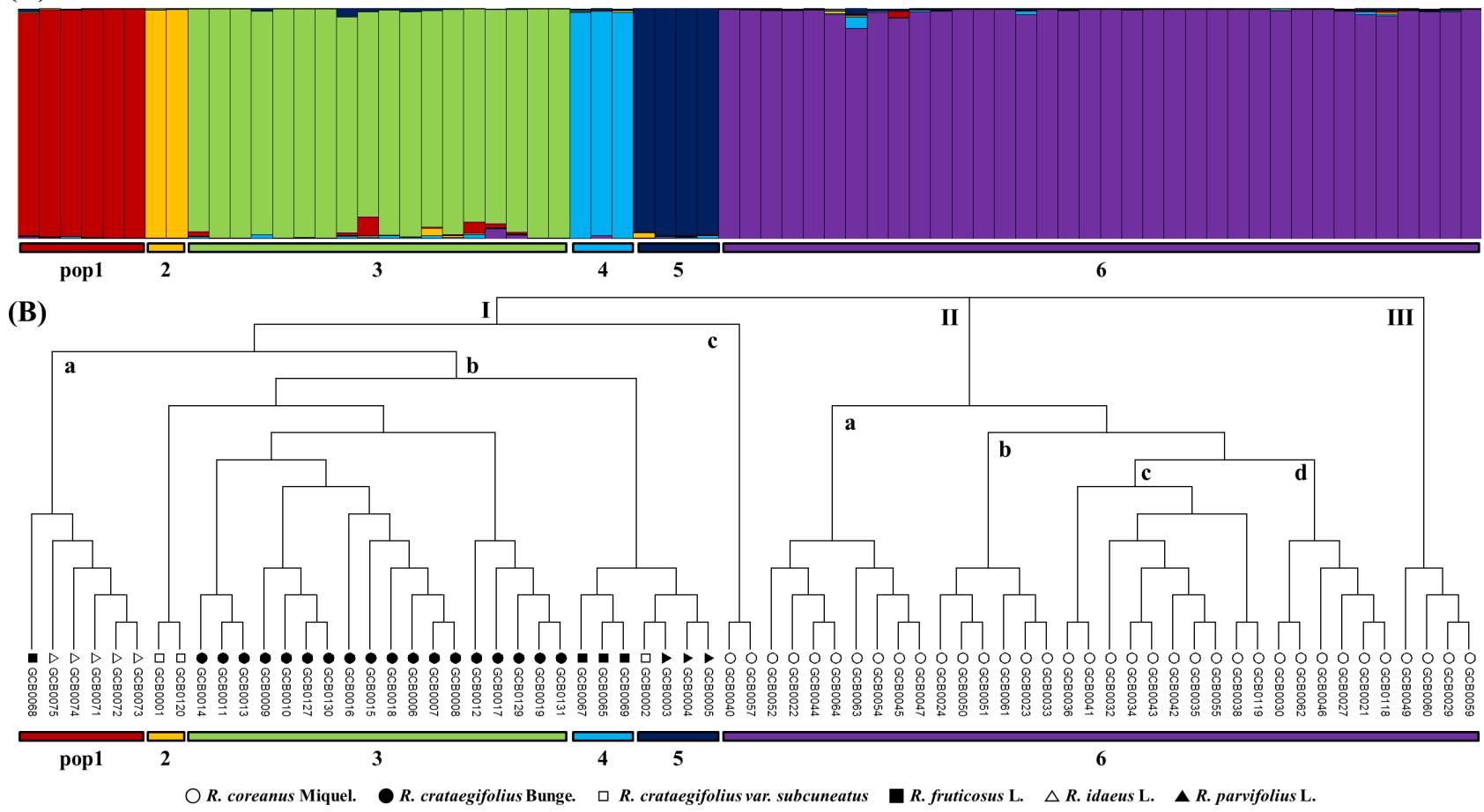

Fig. 2. Model-based population structure (A) and phylogenetic dendrogram (B) based on simple sequence repeat profiles in Rubus species.

pop-4 included three accessions, pop-5 included four accessions, and pop-6 included 36 accessions. Pop-2, pop-3, pop-4, and pop-6 included only one species; $R$. crataegifolius var. subcuneatus, $R$. crataegifolius, $R$. fruticosus, and $R$. coreanus, respectively. Pop-1 included five $R$. idaeus and one $R$. fruticosus accessions, whereas pop-5 contained three $R$. parvifolius and one $R$. crataegifolius var. subcuneatus accessions.

The phylogenetic tree based on a genetic distance matrix could be divided into three major groups (Fig. 2B). Cluster I was further sub-grouped into cluster Ia which contained six Rubus accessions (one R. idaeus L. and five R. idaeus L.), cluster $\mathrm{Ib}$ contained 27 accessions (three $R$. crataegifolius var. subcuneatus, three $R$. fruticosus L., three $R$. parvifolius L., and $18 R$. crataegifolius Bunge.) and cluster Ic contained two accessions ( $R$. coreanus Miquel). Similarly, cluster II was sub-grouped into four clusters; cluster IIa, cluster IIb, cluster IIc and cluster IId that contained eight, six, ten, and six $R$. idaeus $\mathrm{L}$ accessions, respectively. Cluster III contained only 4 accessions (R. idaeus L). Cluster I consisted of six Rubus species, while cluster II and III contained only $R$. coreanus.

\section{DISCUSSION}

Our result revealed a high mean number of alleles per locus (15.3) and a high PIC value (0.76). Dossett et al. (2012) and Marulanda et al. (2012a) reported that the mean number of alleles per locus was 8.6 (range, 2-24) in 146 black raspberry accessions and 4.6 (range, 3-16) in 39 Rubus accessions, respectively. Castillo et al. (2010) showed 7.5 (range, 1-15) alleles in 48 red raspberry accessions and 14.4 (range, 3-29) alleles in 48 blackberry accessions, respectively. Castillo et al. (2010) reported an average PIC value of 0.55 in the red raspberry collection, and Dossett et al. (2012) reported an average PIC value of 0.49 using 21 SSR markers in wild and cultivated black raspberry. These results indicate that the 23 SSR markers acquired from $R$. coreanus might be useful sources for performing genetic studies in Rubus species although it seemed that the higher PIC value obtained in our study was 
due to various Rubus species

The AMOVA analysis revealed that high genetic variability existed among and within Rubus species in the study population. Marulanda et al. (2012a) reported that the variation of Rubus species was mostly attributed to individual variation. Kollmann et al. (2000) concluded that genetic variability in Rubus was determined by the plant propagation system and they demonstrated that there was an effect of cross-pollination between polyploid Rubus species. According to the population structure and phylogenetic analysis based on SSR profiles, the sixty-nine accessions were distinguished by species in a rough way. However, two accessions, GCB0068 ( $R$. fruticosus) and GCB0002 (R. crataegifolius var. subcuneatus), were tied into other Rubus species groups. This result might be caused by the species complexity and weaken species boundaries of geuns Rubus (Thompson 1995; Alice and Campbell 1999).

It is important to evaluate the genetic background of germplasm for the use of genetic resources (Mariac et al. 2006; Tani et al. 2006). Fundamental information on the genetic differences in Rubus species is essential for efficient conservation and management strategies for germplasm. The uncertain diversity of species and artificial groups of the Rubus genus have created confusion with respect to the correct classification of the species at both commercial and scientific levels. The use of these new microsatellite markers will have a major impact on genetic analysis such as GD and evolutionary relationship among Rubus species. The results of this study will provide information for future breeding programs and would be useful for the evaluation and conservation of genetic resources in various Rubus species.

\section{ACKNOWLEDGEMENTS}

This research was supported by a Research Program (Code no. PJ008623) of Agricultural Science and Technology Development funded by National Institute of Agricultural Science, Rural Development Administration, Republic of Korea.

\section{REFERENCES}

Alice LA. 2002. Evolutionary relationships in Rubus (Rosaceae) based on molecular data. International Society for Horticultural Science (ISHS), Leuven, Belgium. pp.79-83.

Alice LA, Campbell CS. 1999. Phylogeny of Rubus (rosaceae) based on nuclear ribosomal DNA internal transcribed spacer region sequences. Am. J. Bot. 86: 81-97.

Castillo NRF, Reed BM, Graham J, Fernández-Fernández F, Bassil NV. 2010. Microsatellite markers for raspberry and blackberry. J. Am. Soc. Hortic. Sci. 135: 271-278.

Dossett M, Bassil NV, Lewers KS, Finn CE. 2012. Genetic diversity in wild and cultivated black raspberry (Rubus occidentalis L.) evaluated by simple sequence repeat markers. Genet. Resour. Crop Evol. 59: 1849-1865.

Evanno G, Regnaut S, Goudet J. 2005. Detecting the number of clusters of individuals using the software STRUCTURE: a simulation study. Mol. Ecol. 14: 2611-2620.

Feingold S, Lloyd J, Norero N, Bonierbale M, Lorenzen J. 2005. Mapping and characterization of new EST-derived microsatellites for potato (Solanum tuberosum L.). Theor. Appl. Genet. 111: 456-466.

Graham J, Smith K, MacKenzie K, Jorgenson L, Hackett C, Powell W. 2004. The construction of a genetic linkage map of red raspberry (Rubus idaeus subsp. idaeus) based on AFLPs, genomic-SSR and EST-SSR markers. Theor. Appl. Genet. 109: 740-749.

Graham J, Squire GR, Marshall B, Harrison RE. 1997. Spatially dependent genetic diversity within and between colonies of wild raspberry Rubus idaeus detected using RAPD markers. Mol. Ecol. 6: 1001-1008.

Jennings DL. 1988. Raspberries and blackberries: their breeding, diseases and growth. Academic Press, London.

Kollmann J, Steinger T, Roy BA. 2000. Evidence of sexuality in European Rubus (Rosaceae) species based on AFLP and allozyme analysis. Am. J. Bot. 87: 1592-1598.

Laurent V, Devaux P, Thiel T, Viard F, Mielordt S, Touzet P, et al. 2007. Comparative effectiveness of sugar beet microsatellite markers isolated from genomic libraries and GenBank ESTs to map the sugar beet genome. Theor. Appl. Genet. 115: 793-805.

Lee GA, Song JY, Choi HR, Chung JW, Jeon YA, Lee JR, et al. 2015. Novel microsatellite markers acquired from 
Rubus coreanus Miq. and cross-amplification in other Rubus species. Molecules 20: 6432-6442.

Lewers KS, Saski CA, Cuthbertson BJ, Henry DC, Staton ME, Main DS, et al. 2008. A blackberry (Rubus L.) expressed sequence tag library for the development of simple sequence repeat markers. BMC Plant Biol. 8: 69.

Liu K, Muse SV. 2005. PowerMarker: an integrated analysis environment for genetic marker analysis. Bioinformatics 21: 2128-2129.

Mariac C, Luong V, Kapran I, Mamadou A, Sagnard F, Deu M, et al. 2006. Diversity of wild and cultivated pearl millet accessions (Pennisetum glaucum [L.] R. Br.) in Niger assessed by microsatellite markers. Theor. Appl. Genet. 114: 49-58.

Marulanda M, Lopez AM, Uribe M. 2012a. Genetic diversity and transferability of rubus microsatellite markers to South American Rubus species. In: M. Caliskan (ed.). The molecular basis of plant genetic diversity. InTech, Rijeka, Croatia.

Marulanda M, López AM, Uribe M. 2012b. Molecular characterization of the Andean blackberry, Rubus glaucus, using SSR markers. Genet. Mol. Res. 11: 322-331.
Peakall R, Smouse PE. 2012. GenAlEx 6.5: genetic analysis in Excel. Population genetic software for teaching and research: an update. Bioinformatics 28: 2537-2539.

Pritchard JK, Stephens M, Donnelly P. 2000. Inference of population structure using multilocus genotype data. Genetics 155: 945-959.

Schuelke M. 2000. An economic method for the fluorescent labeling of PCR fragments. Nat. Biotechnol. 18: 233-234.

Tamura K, Dudley J, Nei M, Kumar S. 2007. MEGA4: Molecular Evolutionary Genetics Analysis (MEGA) software version 4.0. Mol. Biol. Evol. 24: 1596-1599.

Tani N, Yoshimaru H, Kawahara T, Hoshi Y, Nobushima F, Yasui T. 2006. Determination of the genetic structure of remnant Morus boninensis Koidz. trees to establish a conservation program on the Bonin Islands, Japan. BMC Ecol. 6: 14.

Thompson MM. 1995. Chromosome numbers of Rubus cultivars at the National Clonal Germplasm Repository. Hortscience 30: 1453-1456.

Thompson MM. 1997. Survey of chromosome numbers in Rubus (Rosaceae: Rosoideae). Ann. Missouri. Bot. Gard. 84: 128-164. 